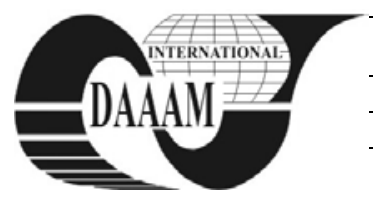

Annals of DAAAM for 2011 \& Proceedings of the 22nd International DAAAM Symposium, Volume 22, No. 1, ISSN 1726-9679 ISBN 978-3-901509-83-4, Editor B. Katalinic, Published by DAAAM International, Vienna, Austria, EU, 2011 Make Harmony between Technology and Nature, and Your Mind will Fly Free as a Bird Annals \& Proceedings of DAAAM International 2011

\title{
OPTIMIZATION OF VALUE ADDED CHAIN IN MANUFACTURING USING STEP
}

\author{
HOFMANN, R[alf]; TOTZAUER, S[oeren]; HAENEL, T[homas] \& WERTHEIM, R[afael]
}

\begin{abstract}
Qualitatively and quantitatively good planning results rely on the experience of the planner and the information available to him. Yet the manifold interfaces along the value added chain result in confusing data redundancy and hinder efficient information usage. This paper will demonstrate how manufacturing information is processed in a process planning system. Single features of rotationally symmetric parts will be combined with machining manufacturing methods regarding requirements and tolerances to be achieved. From these combinations different variants of a manufacturing sequence can be derived via algorithms. These algorithms will be implemented in a specially designed software system. All information from the improved product data model is stored in a database and is easily retrievable for further usage.
\end{abstract}

Key words: product model data, CAP, process planning, machining operation

\section{INTRODUCTION}

At Universities of Chemnitz, Zwickau and Mittweida, an interdisciplinary group of researchers was founded whose research objective is to optimize value-added processes in small and medium enterprises (SMEs) that construct, manufacture and assemble parts and components. Besides, the objective is realizing an advanced feature-oriented product model based on ISO 10303-224 which is applicable at all points along the value-added chain. In this research project, the flow of information transfer is as follows: Feature-based engineering data is read by a specially designed CAD-system and conveyed by a middleware to the assistance system for process planning. All necessary information for generating a process chain as well as the manufacturing sequence (selection of manufacturing resources and calculation of cutting parameters) is processed and stored in a resource database. Then, all planning data is combined with scheduling data delivered from an integrated, external ERP system and finally included into the working schedule by using genetic algorithms. Throughout the self-contained system, starting with construction and ending with manufacturing and assembling, the central information carrier is the ISO standard STEP (standard for the exchange of product model data) which allows for a complete description of the final product. The research focuses on creating an assistance system which is tailored to the individual needs of SMEs working in part and component manufacturing. The system supports and assists technologists. At the same time it documents expertise and provides it in further planning process. Thus, the system is a fast and flexible tool for operational or customer-specific requirements, it helps shortening the planning time and contributes to faster product development.

\section{PRESENTATION OF PRODUCT DEFINITION DATA USING THE ISO 10303 STANDARDS}

The ISO 10303 standards have been continuously developed to ensure the exchange of production data between single elements of the production life cycle (Scheer, 1997). By using a unitary, system-neutral product database (integrated product data model), data exchange problems arising from different CAx formats, versions, systems or from false data conversion can be avoided (Lenders et al., 2007). The ISO 10303 standards are divided into several specifications called application protocols (AP). Each of them is applied to a certain scope (Anderl \& Trippner, 2000). The most well-known STEP interfaces used in CAD formats are AP 203 (Configuration Controlled Design) and AP 214 (Core Data for Automotive Mechanical Design Processes). They can be implemented into modern CAD systems. An advantage is, however, that they can only transmit topological information or only specify a very narrow field of application (free formed surfaces in automotive industry).

This project uses the STEP AP 224 (Mechanical Product Definition for Process Plans Using Machining Features), which was released by the ISO in 2006. It describes a new innovative basis for data exchange (ISO 10303-224, 2006). As in the manufacturing process, this AP uses the design approach of "destruction by machining features". In this approach the final form of every element is achieved by subtracting its shape from the so-called base shape element. In comparison to other APs, it provides a complete product description of the process planning (Fig. 1). Besides geometrical data, the related topology, integration of functional and non-functional requirements for future products, product specific measures and tolerances, description of material and special surface finishing requirements are of significance (Anderl \& Trippner, 2000).

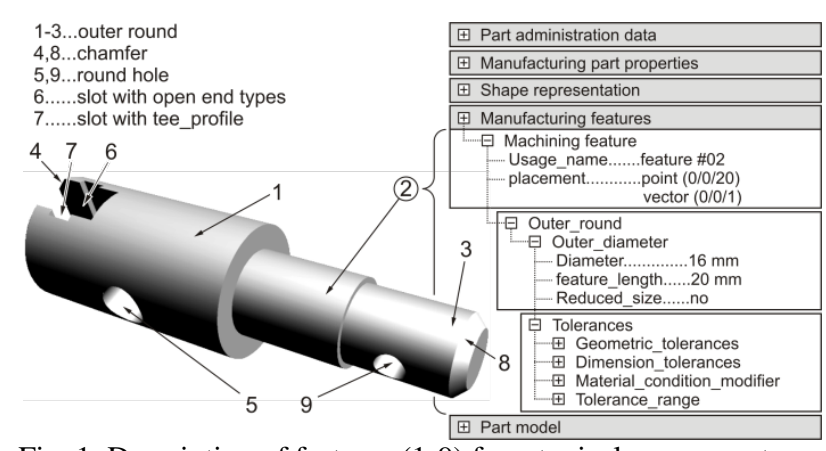

Fig. 1. Description of features (1-9) for a typical component according to IS0 10303-224

\section{DEVELOPMENT OF AN ASSISTANCE PLAN- NING SYSTEM FOR PROCESS PLANNING}

In the feature-based product model (FBPM), components are constructed based on the reference model of the AP 224 and transmitted to an assistance system written in Java. The transmission takes place through a communication layer without interfaces. The assistance system then generates a working plan using data from the FBPM. Job data like scheduling framework (production time), material data, quantity and lot size, is integrated into the planning process in addition to constructionbound data. All further necessary information for planning is 
obtained from a database system, the so-called resource model, containing the resources of a particular SME.

In the first step of process planning, the assistance system is used to select a blank. The attribute "base shape feature" provides the shape of the base body (either cylindrical, block or polygon base shape). Moreover, the FBPM contains further important information, e.g. specifications of material (manufacturing part properties) or order volume (part-model + part + quantity ordered) which influences the production strategy. Since the dimension (LxWxD) of the particular base shape feature only describes the smallest possible body including the part machining and cutting allowances have to be added to the system. After choosing the blank, the features contained in the part are assigned to the machining manufacturing method using a developed matrix based on the ISO 10303-224 reference model and DIN 8580 ff (DIN 8580, 2003). Only machining sequences which achieve the required surface qualities of the particular feature are considered (e.g. predrilling $\rightarrow$ drilling out $\rightarrow$ reaming for tight tolerances).

In the next step of the process planning, the assistance system suggests machining sequences in technological orders. In order to initiate the planning process, all features are categorized into either main form, secondary form or supplementary form (Schiffer \& Tempelhof, 1989) depending on their feature name and certain attributes (e.g. position of the axis of rotation). By categorizing the features, a systematic machining sequence for a finished part of a particular component group can be determined. An example for rotationally symmetric components is shown in Fig. 2.

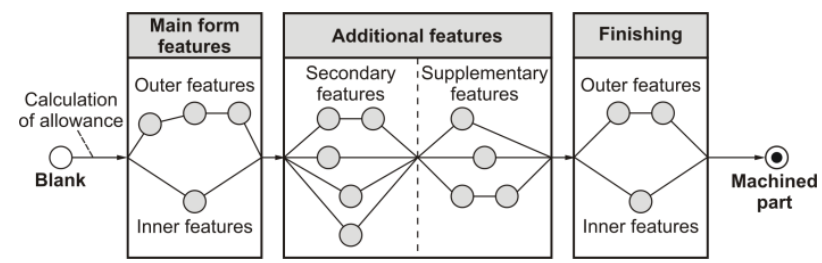

Fig. 2. Technological sequence for rotationally symmetric parts

After categorizing the separate features, elements of the main form are combined and an overall machining strategy for a feature group is determined and machining instructions are specified (see Fig. 3).

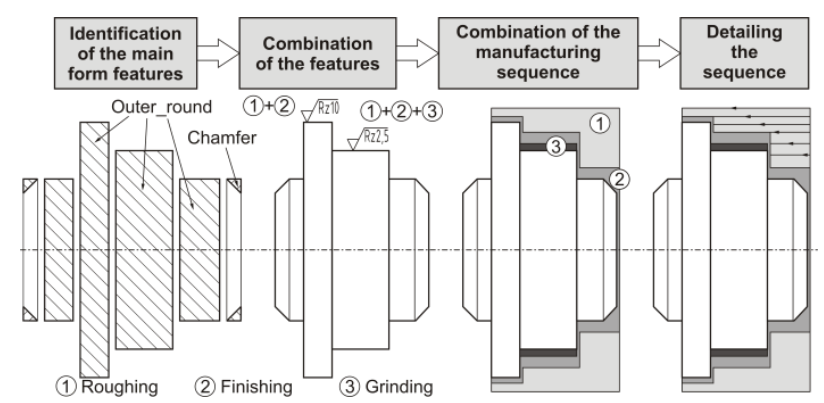

Fig. 3. Planning process for the main form feature group

Analogous to the planning instructions of the main form, the possible workflow for creating secondary forms (slot, hole, pocket, etc.) and supplementary forms (thread, gear, etc.) is determined including use of the manufacturing equipment provided by the resource model. The assistance system supports the user in selecting machines, tools and the manufacturing parameters and predicts the machine load by calculating the estimated cutting forces. This way the assistance system provides the user with information about how his planning decisions affect the process conditions.

The calculated processing durations of the individual manufacturing feature group is an important output of the assistance system for scheduling the complete process chain (related to
Jakobs \& Duerr, 2002). At the end of every planning step, the graphical interface presents the user with a visualization of a network structure consisting of single manufacturing steps from the blank to the manufactured part (see Fig. 2). The nodes represent the features including information about the manufacturing methods and their processing parameters. The respective branches represent independent manufacturing segments and illustrate possible degrees of freedom in manufacturing. Their final sequence can be determined by the user via drag \& drop using information from the scheduling module and ERP data.

\section{CONCLUSION AND PERSPECTIVE}

The assistance system defines the manufacturing variants based on the FBPM using the expertise of the process planer. Machining sequences for typical component assemblies are created by algorithms using a feature $\rightarrow$ manufacturing method matrix. These machining sequences are specified by assigning manufacturing equipment from the resource model. Factors, such as cutting parameters, further dimensions, the expenditure of work regarding the demands on machine load and primary processing time are calculated. The determination of tooling times and expense ratio completes the planning process of a part. Finally, the FBPM including the planning outcome is transmitted to the scheduling module where dispatching and optimization of orders based on ERP data takes place.

The research aim will be a computer system assisting the process planner in every step of the value added chain. Therefore the next step of development will be extending the algorithms from single piece production to assembly production planning and thus researching necessary adjustments to the FBPM. To fully embrace the value-added chain, quality assurance based on ISO 10303-224 shall be integrated in the future as well as adjustment of existing planning data to allow for its usage in the repetitive, variants and similarity process planning.

\section{ACKNOWLEDGEMENTS}

The authors and co-authors appreciate the support of the European Union via the European commission and their European Social Fund (ESF).

\section{REFERENCES}

Anderl, R. \& Trippner, D. (2000). STEP: Standard for the Exchange of Product Model Data, Eine Einfuehrung in die Entwicklung und industrielle Nutzung der Normenreihe ISO 10303 (STEP), B. G. Teubner Verlag, ISBN 3-51906377-8, Leipzig

Degner, W.; Lutze, H. \& Smejkal, E. (2009). Spanende Formung, Theorie, Berechnung, Richtwerte, Hanser Verlag, ISBN 978-3-446-41713-7, Muenchen

Jakobs, H. J. \& Duerr, H. (2002). Entwicklung und Gestaltung von Fertigungsprozessen, Planung und Steuerung der spanenden Teilefertigung, Fachbuchverlag Leipzig, ISBN 3446-21748-7, Leipzig

Lenders, M.; Mueller, J. \& Schuh, G. (2007). PLM mit Modellcharakter, CADplus Business + Engineering 6/2007, WZL RWTH Aachen

Schiffer, F. \& Tempelhof, K. H. (1989). Fertigungsprozessgestaltung im Maschinen- und Geraetebau, VEB Verlag Technik, ISBN 3-341-00195-6, Berlin

ISO 10303-224 (2006). Industrial automation systems and integration - product data representation and exchange part 224, Geneva, Switzerland: International Organization for Standardization (ISO TC 184/SC 4)

DIN 8580 (2003). Fertigungsverfahren - Begriffe, Einteilung, Beuth Verlag, Berlin 